\title{
Diabetes mellitus tipo Il em pessoas idosas: estratégias utilizadas pelos usuários da rede pública de saúde em um município do Sul de Santa Catarina
}

\author{
Karine Tavares Alborghetti", Marie Alexsandra da Silva Oliveira", Maria Regina Silvério"**
}

\section{Resumo}

Trata-se de uma pesquisa de abordagem qualitativa do tipo estudo de caso desenvolvida no segundo semestre de 2011, com o objetivo de compreender o processo de enfrentamento do diabetes mellitus tipo II por pessoas idosas usuárias de uma Unidade de Saúde da Família de um município do Sul de Santa Catarina. A coleta de dados foi realizada no domicílio dos participantes, mediante entrevista semiestruturada. Os dados foram analisados por meio da técnica de análise de conteúdo. Os resultados apontam que as pessoas idosas, diante da condição de serem diabéticas tipo II, apresentam comportamentos favoráveis e não favoráveis ao processo de enfrentamento da doença, que se manifestam por sentimentos de aceitação e conformismo, tristeza e impotência. Na categoria aceitação e conformismo, incluem-se os participantes que aceitam sua condição e convivem com a doença com certa tranquilidade e equilíbrio. Na categoria tristeza e impotência, concentram-se os participantes cuja doença ocasionou mudanças significativas em seu modo de viver, repercutindo de forma negativa em sua qualidade de vida-saúde. Identificou-se que os participantes utilizam as formas de enfrentamento (coping), de modo inter-relacionado, focadas no problema e focadas na emoção, porém, sobressaem as primeiras, as quais se materializam mediante: a adesão ao tratamento, ao controle alimentar e à prática de atividade física; o apoio familiar; a convivência socioafetiva; e a adequação do tratamento às suas condições financeiras. A adesão ao tratamento é a estratégia mais adotada pelos participantes.

Palavras-chave: Pessoa idosa. Diabetes mellitus. Comportamento adaptativo.

* Acadêmica do curso de Enfermagem da Universidade do Sul de Santa Catarina - Campus Tubarão. Endereço para correspondência: Rua do Caçador, 1461, Bairro Caçador, CEP: 88745-000, Capivari de Baixo - SC. E-mail: karyny007@hotmail.com.

** Acadêmica do curso de Enfermagem da Universidade do Sul de Santa Catarina - Campus Tubarão.

**** Professora do curso de Enfermagem da Universidade do Sul de Santa Catarina - Campus Tubarão. Mestra em Educação pela Universidade do Sul de Santa Catarina (Unisul).

$\hookrightarrow$ doi:10.5335/rbceh.2012.019 


\section{Introdução}

Atualmente, o diabetes mellitus (DM) é considerado um problema de saúde pública, sobretudo por se constituir em uma doença crônica que apresenta alta morbidade e mortalidade, com repercussões socioeconômicas significativas. (JANEBRO et al., 2008; DANAEI et al., 2011). Apresenta-se como uma síndrome de evolução crônica cuja prevalência vem crescendo concomitantemente ao processo de industrialização dos últimos anos, segundo Cantos et al. (2004 apud SCHUTZ et al., 2008).

O número de pessoas com a doença aumentou significativamente nos últimos trinta anos em quase todas as partes do mundo. (JANEBRO et al., 2008; DANAEI et al., 2011). Estes autores salientam que o número de diabéticos é marcantemente maior do que as projeções divulgadas, as quais estimam 285 milhões em todo o planeta. Eles constataram que existem, no mundo, 347 milhões de diabéticos, dos quais 138 milhões vivem na China e na Índia e 36 milhões, nos Estados Unidos e na Rússia. Nesse contexto, o Brasil ocupa a $119^{a}$ posição, com o índice de 20 milhões de pessoas com a enfermidade (2011).

A incidência de DM, nas últimas décadas, vem aumentando gradativamente, em decorrência de um estilo de vida inadequado, especialmente da má alimentação e do sendentarismo. (PIARETE; FERREIRA, 2008).

Conforme dados do Sistema de Informações da Atenção Básica (Siab), estão cadastrados no estado de SC, nos mais diversos municípios, 85.417 indivíduos com diabetes, dos quais 716 vivem no município de Capivari de Baixo - SC, local sítio do presente estudo. (BRASIL, 2011a).

Segundo a Sociedade Brasileira de Diabetes (SBD, 2009), o DM compreende um grupo de doenças metabólicas caracterizado por um aumento anormal de glicose no sangue devido à deficiência de produção da insulina.

De acordo com Rodrigues, Szymaniak e Sobrinho (2010), dentre os tipos de DM, destacam-se os grupos I e II. O desenvolvimento de diabetes mellitus tipo I (DMI) resulta da destruição das células pancreáticas, com deficiência na produção de insulina. $\mathrm{O}$ diabetes mellitus tipo II (DMII) é caracterizado por um estado de resistência à ação da insulina associado a uma relativa deficiência de sua secreção.

Da mesma forma, a SBD descreve os dois tipos de diabetes. O tipo I, que se caracteriza como doença crônica, pode acometer indivíduos de diferentes faixas etárias, porém atinge comumente crianças, adolescentes e adultos jovens. Essa doença caracteriza-se pela destruição das células beta do pâncreas, resultante da deficiência completa de insulina. $\mathrm{O}$ tipo II é ocasionado por defeitos relacionados à secreção e à ação da insulina. A doença pode acometer indivíduos em qualquer faixa etária, porém, é mais frequente em adultos acima de 40 anos de idade (SBD, 2009).

Trata-se de uma doença global, insidiosa, que decorre de vários fatores e sobremaneira, trazendo consequentes alterações ao cotidiano das pessoas e afetando a sua qualidade e a sua ex- 
pectativa de vida. (RIBEIRO; ROCHA; POPIM, 2010). Na visão dos mesmos autores, a forma mais frequente de diabetes na pessoa idosa é o tipo II, o qual se manifesta pela elevação dos níveis glicêmicos, comprometendo vários órgãos e sistemas, especialmente coração, olhos, rins e sistema nervoso. O aumento de gordura no sangue pode acarretar problemas cardiovasculares como hipertensão, aterosclerose e infarto do miocárdio, o que interfere na funcionalidade física, psicológica e social do indivíduo.

Ainda segundo a SBD (2011), o diabetes acomete $18 \%$ da população com mais de 60 anos de idade, e, do total, $50 \%$ apresentam o tipo II. Essa modalidade representa, para esse grupo etário, maior risco de morte prematura e associação com outras doenças. Por sua vez, Reis et al. (2009) destacam que o envelhecimento populacional e o aumento da expectativa de vida coexistem com as doenças crônicas não transmissíveis, dentre as quais, o diabetes. Certamente, isso pode ser visto como prerrogativa ao aumento da incidência da doença entre a geração idosa.

Referentemente ao controle e à prevenção de agravos e sequelas, Piarete e Ferreira (2008) salientam que as complicações do diabetes, quando devidamente controladas, podem ser evitadas; porém, faz-se necessário que o indivíduo portador aceite a doença e concretize a adesão ao tratamento.

No tocante à atenção à saúde da pessoa idosa, Ribeiro, Rocha e Popim (2010) alertam que o crescente aumento de indivíduos diabéticos acima de 60 anos é um fenômeno universal que impõe a necessidade de políticas públicas capazes de atender eficazmente essa parcela da população.

Na opinião de Rodrigues et al. (2009), é fundamental buscar estratégias educativas que ampliem o conhecimento do portador de diabetes mellitus e que repercutam favoravelmente no seu convívio com a doença.

Partindo-se do pressuposto de que o diabetes consiste em uma doença crônica presente entre as pessoas que envelhecem e de que é útil conhecer os seus sentidos e significados para esses indivíduos, considerou-se pertinente desenvolver um estudo capaz de apreender o processo de enfrentamento dessa patologia pelos usuários do serviço público de saúde. Além disso, o fato de as estimativas predizerem que, em 2025, o número de pessoas com diabetes chegará próximo aos 380 milhões (BRASIL, 2011b) reforça a importância de estudos sobre a temática. Assim sendo, selecionou-se como amostra dessa população os idosos usuários de uma Unidade de Saúde da Família do município de Capivari de Baixo - SC.

Diante desse contexto, delimitou-se a seguinte questão norteadora: como pessoas idosas usuárias de uma Unidade de Saúde da Família do município de Capivari de Baixo - SC enfrentam o diabetes tipo II?

Como objetivo do estudo, delineou-se o seguinte: conhecer as formas de enfrentamento do diabetes mellitus tipo II por pessoas idosas usuárias de uma Unidade de Saúde da Família do município de Capivari de Baixo - SC. 


\section{Referencial teórico}

A teoria do enfrentamento, ou estratégia de coping, proposta por Lazarus e Folkman (1984), foi a matriz teórica utilizada na reflexão. A estratégia, de acordo com os autores, consiste em um conjunto de ações, comportamentos ou pensamentos que as pessoas utilizam para lidar com o estressor. Trata-se de um processo dinâmico que permite a avaliação e a reavaliação contínua da relação pessoa-ambiente, o que pode resultar em situações melhores ou piores do que a inicial. (LAZARUS; FOLKMAN, 1984 apud VIVIAN; ARGIMON, 2009).

Existem duas categorias funcionais de coping, "o coping focalizado no problema e o coping focalizado na emoção". A focalizada na emoção é aquela em que os indivíduos buscam estratégias para evitar os - ou se afastar dos - eventos estressores, ou, então, para conviver com a situação de forma mais positiva, reduzindo o impacto emocional do estressor. A categoria focalizada no problema é aquela em que as pessoas identificam o problema que estão vivenciando e buscam "soluções alternativas, optando entre elas". (LAZARUS; FOLKMAN, 1984 apud RODRIGUES; CHAVES, 2008).

Trentini et al. (2005), com base em Lazarus e Folkman (1991), consideram que essas categorias se inter-relacionam, posto que as pessoas, quando se deparam com um evento estressor, utilizam ambas as categorias de enfrentamento. Uma estratégia focada inicialmente no problema pode, também, focar-se na emoção. Assim, essas estratégias atuam de forma dinâmica e recíproca.
Por sua vez, Correia (2010), igualmente a partir de Lazarus e Folkman (1980), destaca que o modo como cada pessoa utiliza as estratégias de coping depende da avaliação que ela faz acerca da situação vivenciada. Nessa perspectiva, o diagnóstico impõe aos indivíduos portadores da doença uma série de desafios, na medida em que lhes exige adaptações nas diversas dimensões (física, psicológica e social) de forma que se amenizem as ameaças ao seu bem-estar. (AMORIM; COELHO, 2008; CORREIA, 2010).

Diante do exposto, considerou-se a teoria do enfrentamento ou estratégia de coping apropriada para a compreensão da realidade que se tomou como objeto de estudo.

\section{Metodologia}

Trata-se de uma pesquisa de natureza qualitativa do tipo estudo de caso. A abordagem qualitativa trabalha com objetos de estudo que não podem ou não devem ser quantificados, tais como significados, aspirações, crenças, valores e atitudes. (MINAYO, 2007). Segundo Lüdke e André, no estudo de caso, "o objeto estudado é tratado como único, uma representação singular da realidade que é multidimensional e historicamente situada”. (1986, p. 21).

Participaram do estudo sete pessoas idosas portadoras de DM tipo II, selecionadas pelas pesquisadoras nos cadastros dos usuários de uma Unidade de Saúde da Família do município de Capivari de Baixo - SC. O número de participantes foi definido com base nos 
seguintes critérios de inclusão: pessoas idosas de ambos os sexos, na faixa etária entre 60 e 85 anos, com algum tipo de limitação decorrente da doença, que não apresentassem deficit cognitivo e que aceitasse participar do estudo de forma espontânea, assinando o Termo de Consentimento Livre e Esclarecido (TCLE).

Quanto aos aspectos éticos, foram observados, no desenvolvimento do estudo, os preceitos da Resolução 196/96 do Conselho Nacional de Saúde (BRASIL, 1996), que trata das diretrizes de pesquisas com seres humanos, a saber: autorização da instituição para a realização da pesquisa; assinatura do TCLE pelos participantes do estudo de maneira espontânea; esclarecimento aos participantes sobre os objetivos e os métodos adotados na realização da pesquisa; respeito ao anonimato e à privacidade dos participantes da pesquisa; respeito às crenças e aos valores dos participantes da pesquisa; garantia aos participantes do estudo da liberdade de participarem ou desistirem da pesquisa a qualquer momento, sem que isso lhes ocasionasse prejuízo de qualquer natureza; respeito ao direito dos participantes de esclarecimentos antes, durante e após o curso da pesquisa; acordo quanto ao horário da entrevista com os participantes do estudo, a fim de não interferir em sua rotina diária; garantia do sigilo das informações obtidas; divulgação dos dados do estudo sem distorção e devolução dos dados à instituição pesquisada.

$\mathrm{O}$ projeto foi encaminhado para a apreciação do Comitê de Ética em Pesquisa (CEP) da Universidade do Sul de Santa Catarina, obtendo sua aprovação, conforme Parecer n. 11.406.4.04.III.
A fim de assegurar o seu anonimato, os participantes foram nomeados no estudo através de pseudônimos que designam sentimentos: emoção, confiança, otimismo, perseverança, segurança, esperança e afeto.

Os dados foram coletados por meio de entrevista semiestruturada, realizada nos domicílios dos participantes. $\mathrm{O}$ instrumento de entrevista conteve duas partes: a primeira parte tratou de informações pessoais dos participantes (gênero, idade, estado civil, escolaridade, profissão, renda familiar, número de pessoas que residiam no domicílio, tempo decorrido a partir do diagnóstico da doença e complicações decorrentes da doença); a segunda foi composta por duas questões orientadoras voltadas à convivência com o diabetes mellitus tipo II e às medidas de enfrentamento da doença.

Para a realização das entrevistas, as pesquisadoras estabeleceram um primeiro contato com as pessoas idosas pré-selecionadas, acompanhadas da agente comunitária de saúde responsável pela microárea, com o intuito de prestarem esclarecimentos sobre a pesquisa e lhes convidarem a participar, bem como para agendar a visita para a coleta de dados.

Os dados foram registrados durante a entrevista no instrumento de pesquisa. Ao final da entrevista, as pesquisadoras procederam à leitura das informações registradas, com vistas a possibilitar aos entrevistados a inclusão ou exclusão de alguma informação, caso o desejassem. Esses dados foram analisados com base no método de análise de conteúdo proposto por Gomes (2007), compreendendo: a) leitura compreensiva do material 
selecionado, com o intuito de obter-se a visão do conjunto e das especificidades das informações, para se construir uma estrutura de base para a interpretação; b) exploração do material, desenvolvida a partir da decomposição das ideias explícitas e implícitas contidas nos depoimentos dos entrevistados, a fim de se problematizar os pensamentos em busca de sentidos mais amplos; c) elaboração de síntese interpretativa, por meio da articulação entre o conjunto das ideias obtidas, os objetivos e o referencial teórico do estudo.

\section{Resultados e discussão}

\section{I - Caracterização dos participantes do estudo}

Participaram do estudo 7 pessoas idosas com DM tipo II. Destas, 2 eram do sexo feminino e 5 do sexo masculino, com idade entre 60 e 85 anos, prevalecendo a faixa etária entre 65 e 75 anos. Dos participantes, 4 eram casados e 3 viúvos. Três cursaram o ensino fundamental e 4, o ensino médio. Quanto à profissão, 2 eram do lar e 5 eram aposentados, com renda familiar entre 2 e 4 salários mínimos. O número de familiares que residiam no domicílio variou entre 2 e 3 pessoas.

O tempo decorrido a partir do diagnóstico da doença variou entre 5 e 40 anos, prevalecendo o tempo médio de 20 anos. Foram referidos como complicações decorrentes do DM II o comprometimento da visão e problemas vasculares, os quais ocasionaram a necessidade de amputação de membros inferiores.
II - Sentimentos apresentados pelos idosos em relação à doença e suas estratégias de enfrentamento, sob a ótica da teoria coping

Das informações acerca da condição de ser diabético tipo II, evidenciam-se sentimentos favoráveis e não favoráveis ao enfrentamento da doença. Os relatos foram agrupados em duas categorias de análise: "aceitação - conformismo" e "tristeza - impotência".

No que diz respeito à categoria aceitação - conformismo, alguns depoimentos sinalizam aceitação e convivência com o diabetes II com base em certa tranquilidade e equilíbrio.

Tem que se adaptar, [...] não adianta esquentar a cabeça, depois que tem é preciso aceitar (Segurança).

Normal, a gente não pode fazer nada, tem que fazer o melhor que a gente puder (Perseverança).

Há pessoas que convivem com determinadas doenças e mostram-se dispostas a enfrentá-las. Justin (1988 apud CORREIA, 2010) informa que as estratégias de enfrentamento (coping) em relação ao processo de saúde - adoecimento humano apresentam vários benefícios, sendo importante adquirir conhecimentos acerca de estratégias que possam modificar o estresse associado ao problema de saúde.

Francioni e Silva (2004 apud FRANCIONI; SILVA, 2007) referem que é possível ter qualidade de vida mesmo sendo portador de doença crônica, desde que a pessoa conheça seus limites e as possibilidades dentro das quais possa se expressar e criar novas formas de lidar com a doença. Por isso, é fundamental aprender a ter um comportamento de 
autocontrole, definir metas e construir caminhos que promovam a qualidade de vida.

A categoria tristeza - impotência revela que, para alguns participantes do estudo, o DM II ocasionou mudanças significativas em seu modo de viver, repercutindo de forma negativa em sua qualidade de vida-saúde.

[...] tenho um desânimo porque tudo que eu gosto eu não posso. Eu sou apaixonada por doçura, tem dia que eu fico louca para comer pelo menos uma balinha, e não posso (Esperança).

A gente se sente "terminado", sou uma pessoa hoje em dia mais triste. Não posso nem viajar, a visão é ruim, as pernas já não dão mais, já tive dois dedos amputados (Otimis$\mathrm{mo})$.

Conforme os depoimentos, a proibição de ingestão de alimentos que contêm açúcares e as limitações físicas decorrentes do DM, associadas a outras limitações decorrentes da idade, são aspectos que geram grande desconforto.

Para além dessas questões, alguns participantes externaram sentimentos negativos a respeito da doença, ao responsabilizá-la pelo desencadeamento de episódios depressivos.

Me sinto mal, ruim; ela complica tudo, só choro, não tenho vontade de fazer nada, fico assim jogada no sofá ou na cama (Emoção).

Tenho uma tristeza muito grande. Me sinto triste de saber que eu tenho diabetes, me desanimei e tive até depressão, minha visão piorou, tô indo sempre no médico oculista para tratar o glaucoma (Esperança).

Essas situações vêm ao encontro do pensamento de Marcelino e Carvalho
(2005), os quais comentam que, ao adoecer, o portador de diabetes vivencia inúmeros sentimentos negativos, tais como medo, insegurança, tristeza e desânimo.

Desse modo, as estratégias de enfrentamento (coping) adotadas pelas pessoas idosas com diabetes materializaram-se por meio de ações que foram agrupadas nas seguintes categorias de análise: adesão ao tratamento; atitudes em relação à alimentação e prática de atividade física; apoio familiar e convivência afetivo-social; e condições financeiras.

Essas estratégias têm como função administrar ou alterar o estressor. Nesse cenário, o DM II, por ser uma doença de manejo complexo, impõe ao grupo etário em pauta uma postura disciplinar em constante vigilância, o que, de certa forma, atribui mudanças ao estilo de vida habitual (hábitos alimentares e sociais, além de terapêutica medicamentosa).

A adesão ao tratamento foi uma das estratégias de enfrentamento que sobressaiu nos discursos dos investigados.

Faço o tratamento certinho, me cuido e quando extrapolo já meço e faço insulina (Otimismo).

Tomo medicação todo dia, eu mesmo sei que não posso fazer bobagem (Perseverança).

Gimenes, Zanetti e Haas (2009) consideram que a adesão ao tratamento é um fenômeno complexo que pode ser influenciado por diversos fatores, com destaque para a confiança que o paciente deposita na terapia medicamentosa, com base nas informações recebidas no espaço da Unidade de Saúde, no curso das consultas. 
Silvestre et al. (2008) ressaltam que as estratégias de enfrentamento (coping) contribuem para que o indivíduo se adapte às situações de estresse. Para que isso aconteça, porém, a pessoa necessita crer na mudança e nos benefícios que essas estratégias lhe trarão.

No tocante à categoria atitudes em relação à alimentação, identificou-se que há posturas diferenciadas quanto ao seguimento da dieta.

Como aquilo que é possível comer, me cuido (Otimismo).

Com a alimentação não estou me cuidando, como coisas que não eram [sic] para comer (Emoção).

Pontieri e Bachion (2010) consideram que os hábitos alimentares são adquiridos ao longo da vida e são influenciados por diversos fatores. Manter hábitos esses saudáveis é uma condição essencial a indivíduos com diabetes.

Para Curcio, Lima e Alexandre (2011), os indivíduos portadores de DM necessitam aprender a conviver, durante toda a vida, com uma doença cujas complicações clínicas prejudicam sobremaneira sua saúde, devendo enfrentar mudanças significativas em seu estilo de vida, especialmente no tocante aos hábitos alimentares e à atividade física/ prática corporal, como também à adesão à terapia medicamentosa (insulinas, hipoglicemiantes orais).

A atividade física regular é um importante aliado ao plano terapêutico do idoso/da idosa com diabetes tipo II, uma vez que controla os níveis glicêmicos e contribui para a promoção da saúde como um todo, ou seja, nos planos físico e mental.

Identificou-se que apenas alguns dos participantes praticam atividade física regularmente.

Caminho todo dia (Esperança).

Faço caminhada de 20 a 30 minutos todo dia (Otimismo).

Lemos (2008), com base em Dullios e na International Diabetes Federation (2006), salienta que a prática de atividade física deve ser adotada de forma regular pelos diabéticos, de acordo com sua capacidade. Esse tipo de prática contribui para o controle glicêmico e para a promoção da sua qualidade de vida.

Todavia, a maioria dos sujeitos do estudo não pratica atividades físicas, devido às complicações decorrentes da doença.

Não faço nenhuma atividade física, depois que amputei minha perna não pude fazer mais caminhada (Confiança).

A atividade que eu faço é caminhar na cozinha, no quintal, me levanto da cama, mas caminhar como eu caminhava, isso eu não faço mais (Perseverança).

Por seu turno, Berlese, Moreira e Sanfelice (2007) mencionam que a inatividade física tem uma forte relação com o desenvolvimento de doenças crônicas, inclusive o diabetes mellitus tipo II. A amputação de algum membro ou de parte deste é uma das repercussões da evolução do DM II, a qual compromete significativamente a qualidade de vida - saúde dos portadores dessa doença.

Salomé, Espósito e Silva (2008) referem que a amputação, para a pessoa portadora de diabetes, extrapola a 
perda dos membros ou de parte deles. $\mathrm{O}$ evento ocasiona mudanças significativas na vida do indivíduo, sobretudo com a diminuição da autoestima e a piora da qualidade de vida, condição que requer um processo de adaptação, sendo necessário o apoio familiar e social.

$\mathrm{O}$ apoio familiar e a preservação da vida afetivo-social são, pois, estratégias que favorecem o enfrentamento da doença pelas pessoas idosas pesquisadas.

\begin{abstract}
A minha família fica preocupada quando o meu diabetes aumenta, eles estão sempre comigo, me ajudando em tudo o que eu preciso (Segurança).

Eles cuidam muito de mim e se preocupam se eu estou precisando de alguma coisa, estão sempre me visitando (Otimismo).
\end{abstract}

Santos et al. (2011) confirmam que o suporte familiar contribui para que $o$ indivíduo enfrente as adversidades decorrentes da doença. Em contrapartida, a ausência desse tipo de suporte é marcante para o portador de DM II, posto que a necessidade de enfrentar diversos desafios o deixa mais fragilizado.

Para um dos participantes do estudo, a falta de apoio o leva a sentir-se solitário e carente.

[...] a gente fica mais sozinho, os filhos vivem para si; agora estou morando com essa filha porque ela me convidou para morar com ela (Perseverança).

De acordo com Santos et al. (2011), a pessoa com diabetes mellitus nem sempre recebe o apoio familiar indicado para enfrentar a doença e ela própria passa a demonstrar a necessidade de aproximação e suporte da família.
Os problemas ocasionados pelo diabetes extrapolam a dimensão físico-emocional e podem comprometer, também, a qualidade das relações afetivo-sociais daqueles que têm a doença. No entanto, identificou-se que alguns participantes deste estudo mantêm vida social ativa.

Eu passeio muito, saio no final de semana para dançar, participo do clube de mães, adoro ir no [sic] bingo (Esperança).

Eu passeio bastante, gosto muito de dançar, estou até procurando uma namorada; vou aos bailes da terceira idade e tenho muitos amigos lá (Segurança).

Outros participantes, por sua vez, vivenciam situações de exclusão e isolamento:

Não saio de casa para nada, quando preciso consultar, a médica vem aqui me ver; só fico na cama e, às vezes, ando pelo quintal. Não tenho com quem conversar, só com essa filha que [sic] eu moro e quando passa um conhecido na rua eu converso um pouquinho (Perseverança).

Não saio muito de casa, só mesmo para consultar, pegar remédio e fazer voltas da casa (Afeto).

Péres et al. (2007) destacam que o convívio social da pessoa com diabetes pode interferir no seu equilíbrio orgânico, e essa interferência, muitas vezes, extrapola a dimensão individual, a ponto de repercutir na vida familiar e social.

Nunes (2005) corrobora o exposto, ao mencionar que o diabetes é uma doença crônica que pode desencadear restrições nas áreas físicas e sociais e, com isso, acarretar mudanças significativas na vida do portador. 
Outro aspecto a ser enfrentado pela pessoa idosa diabética diz respeito aos recursos financeiros para as despesas com terapêutica medicamentosa, exames e alimentação dietética. Essas despesas interferem na adesão ao tratamento, uma vez que, dependendo das condições de cada um, podem ser elevadas, considerando-se que nem todos os medicamentos e exames necessários são fornecidos pelo serviço público de saúde.

Tem remédio que é caro, até tive que voltar para o Posto para o médico trocar a minha medicação (Emoção).

Tenho despesas com médicos, exames, medicamentos (Otimismo).

Santos (2010) aponta essa situação, ao considerar que as condições socioeconômicas da pessoa portadora de diabetes influenciam de forma negativa em seu tratamento, na medida em que a doença requer gastos com a alimentação e com medicamentos que nem sempre são encontrados nas unidades da rede pública de saúde.

\section{Considerações finais}

Este estudo revelou que as pessoas idosas com diabetes tipo II apresentam comportamentos favoráveis e não favoráveis ao processo de enfrentamento da doença, os quais se manifestam por meio de sentimentos de aceitação e conformismo, tristeza e impotência.

A categoria aceitação e conformismo diz respeito aos participantes que aceitam e convivem com a doença com certa tranquilidade e equilíbrio. A categoria tristeza e impotência refere-se aos participantes para os quais a doença oca- sionou mudanças significativas no modo de viver, repercutindo de forma negativa em sua qualidade de vida-saúde. Para alguns participantes, a doença tem sido responsável pelo desencadeamento de episódios depressivos.

Quanto às estratégias de enfrentamento (coping), identificou-se que os participantes as utilizam de forma inter-relacionada, ou seja, utilizam tanto as estratégias focadas no problema como as focadas na emoção, prevalecendo as primeiras. Tais estratégias materializam-se por meio da adesão ao tratamento, de atitudes alimentares, da prática de atividade física, do apoio familiar, da convivência afetivo-social e da adequação do tratamento às suas condições financeiras. A adesão ao tratamento foi a estratégia mais referida pelos participantes, ao passo que as demais que se alinham ao âmbito da promoção de saúde e qualidade de vida, ainda não são adotadas de modo sistemático e contínuo.

O DM constitui um problema de saúde pública, em vista de sua alta incidência e de sua condição de doença crônica, que apresenta alta morbidade e mortalidade, além de repercussões importantes sobre a qualidade de vida do seu portador, especialmente dos idosos. Em vista disso, evidenciou-se que se faz necessária uma intensificação das campanhas de educação para a saúde, com abordagem específica dessa realidade, não apenas por parte dos serviços de saúde como também de todos os órgãos com poder de abrangência e de comunicação com a população. 
Diabetes mellitus type II in elderly: strategies used by users of heath public network in a city on south of Santa Catarina

\section{Abstract}

Qualitative approaching search type case study developed on the second semester of 2011 aiming the comprehension of the facing process of diabetes mellitus type II by the elderly users of a Family Health Unity of a city in south of Santa Catarina - SC. The data collection was made at the participants' home through a semi-structured interview. The data was analyzed by a technique of content analysis. The results pointed that the elderly, in front of the condition of DM II carriers, they present favorable and not favorable behaviors to the facing process of the disease, which is manifested through the feelings of acceptance and conformity, sadness and impotence. On the acceptance and conformity category, include the participants that accept and live with the disease with a certain tranquility and balance. On the sadness and impotence category, include the participants whose the disease occurred significant changes on their way of life that rebound a negative form in their life-health quality. Was identified that the participants used the facing forms (coping) with an interrelated way focused on the problem and focused on the emotion, but the firsts emerge. These are materialized through the treatment acceptation, with eating control, with practice of a physical exercise, with family support, with socioaffective living and the suiting of the treatment to the financial condition of the patient. The acceptation of the treatment is the most adopted strategy among the patients.

Keywords: Elderly. Diabetes mellitus. Adaptive behavior.

\section{Referências}

AMORIM, I. L.; COELHO, R. Diabetes mellitus tipo 2 e sintomas psicopatológicos. Psicologia, Saúde \& Doenças, Lisboa, v. 9, n. 2, p. 319-333, ago. 2008.

BERLESE, D. B.; MOREIRA, M. C.; SANFELICE, G. R. A importância do exercício físico e sua relação com diabetes mellitus tipo 2. Revista Digital, Buenos Aires, n. 115, dez. 2007.

BRASIL. Ministério da Saúde. Conselho Nacional de Saúde. Resolução 196, de 10 de outubro de 1996. Aprova diretrizes e normas regulamentadoras de pesquisas envolvendo seres humanos. Disponível em: <http://www. ufrgs.br/bioetica/res19696.htm>. Acesso em: 05 ago. 2011.

do diabetes. 2011a.

.Apresentação: dia mundial

Disponível em: <http://portal.saude.gov.br/>. Acesso em: 05 ago. 2011.

. Datasus: Sistema de Infor-

mação de Atenção Básica. 2011b. Disponível em: <http://tabnet.datasus.gov.br/>. Acesso em: 05 ago. 2011.

CORREIA, A. C. R. Coping e autoeficácia em pais de crianças e adolescentes com diabetes tipo 1. 2010. 83 f. Dissertação (Mestrado em Psicologia Clínica e da Saúde) - Universidade Fernando Pessoa, Porto, 2010.

CURCIO, R.; LIMA, M. H. M.; ALEXANDRE, N. M. C. Instrumentos relacionados ao diabetes mellitus adaptados e validados para a cultura brasileira. Revista Eletrônica de Enfermagem, Goiás, v. 13, n. 2, p. 331-337, abr./jun. 2011.

DANAEI, G. et al. National, regional, and global trends in fasting plasma glucose and diabetes prevalence since 1980: systematic analysis of health examination surveys and epidemiological studies with 370 countryyears and 2.7 million participants. The Lancet, Boston, v. 378, n. 2, p. 35, jul. 2011. 
FRANCIONI, F. F.; SILVA, D. G. V. O processo de viver saudável de pessoas com diabetes mellitus através de um grupo de convivência. Texto \& Contexto - Enfermagem, Florianópolis, v. 16, n. 1, p. 105-111, jan./mar. 2007. GIMENES, H. T.; ZANETTI, M. L.; HAAS, V. J. Fatores relacionados à adesão do paciente diabético à terapêutica medicamentosa. Revista Latino-Americana de Enfermagem, Ribeirão Preto, v. 17, n. 1, p. 46-51, jan./fev. 2009.

GOMES, R. Análise e interpretação de dados de pesquisa qualitativa. In: MINAYO, M. C. S.; DESLANDES, S. F.; GOMES, R. Pesquisa social: teoria, método e criatividade. 26. ed. Petrópolis, RJ: Vozes, 2007. p. 79-108.

JANEBRO, D. I. et al. Efeito da farinha da casca do maracujá-amarelo (Passiftora edulis f. flavicarpa Deg.) nos níveis glicêmicos e lipídicos de pacientes diabéticos tipo 2. Revista Brasileira de Farmacognosia, João Pessoa, v. 18, supl. 0, p. 724-732, dez. 2008.

LAZARUS, R. S.; FOLKMAN, S. Stress, appraisal and coping. New York: Springer Publishing Company, 1984.

LEMOS, S. S. Fatores que interferem na qualidade de vida com diabetes: opinião dos portadores. 2008. 116 f. Dissertação (Mestrado em Ciências da Saúde) - Universidade de Brasília, Brasília, 2008.

LÜDKE, M.; ANDRÉ, M. E. D. A. Abordagens qualitativas de pesquisa: a pesquisa etnográfica e o estudo de caso. São Paulo: EPU, 1986.

MARCELINO, D. B.; CARVALHO, M. D. B. Reflexão sobre o diabetes tipo 1 e sua relação com o emocional. Psicologia: Reflexão $e$ Crítica, Maringá, v. 18, n. 1, p. 72-77, 2005.

MINAYO, M. C. S. O desafio do conhecimento: pesquisa qualitativa em saúde. 10. ed. São Paulo: Hucitec, 2007.

NUNES, M. Apoio social na diabetes. Revista Millenium, Viseu, s. v., n. 31, p. 135-149, maio 2005. Disponível em: <http://www.ipv. $\mathrm{pt} / \mathrm{millenium} / \mathrm{millenium} 31 / 10$.pdf $>$. Acesso em: 02 nov. 2011.
PÉRES, D. S. et al. Dificuldades dos pacientes diabéticos para o controle da doença: sentimentos e comportamentos. Revista Latino-Americana de Enfermagem, Ribeirão Preto, v. 15, n. 6, p. 1105-1112, nov./dez. 2007.

PIARETE, D.; FERREIRA, K. C. O processo de adaptação do portador de diabetes mellitus tipo 2, após constatação diagnóstica: a busca por sentido. 2008. $124 \mathrm{f}$. Monografia (Graduação em Psicologia) - Universidade Anhanguera, Santo André, SP, 2008.

PONTIERI, F. M.; BACHION, M. M. Crenças de pacientes diabéticos acerca da terapia nutricional e sua influência na adesão ao tratamento. Ciência e Saúde Coletiva, Rio de Janeiro, v. 15, n. 1, p. 151-160, jan. 2010.

REIS, L. A. et al. Avaliação da qualidade de vida em idosos portadores de diabetes mellitus tipo 2. C\&D-Revista Eletrônica da Fainor, Vitória da Conquista, v. 2, n. 1, p. 64-76, jan./dez. 2009.

RIBEIRO, J. P.; ROCHA, S. A.; POPIM, R. C. Compreendendo o significado de qualidade de vida segundo idosos portadores de diabetes mellitus tipo II. Escola de Enfermagem Anna Nery, Botucatu, v. 14, n. 4, p. 765-771, out./dez. 2010.

RODRIGUES, A. B.; CHAVES, E. C. Fatores estressantes e estratégias de coping dos enfermeiros atuantes em oncologia. Revista Latino-Americana de Enfermagem, São Paulo, v. 16 , n. 1, p. 24-28, jan./fev. 2008.

RODRIGUES, A. N.; SZYMANIAK, N. P.; SOBRINHO, J. A. Influência das dermatoses na qualidade de vida do portador de diabetes mellitus. Ciência \& Saúde Coletiva, Rio de Janeiro, v. 15, supl. 1, p. 1325-1332, jun. 2010.

RODRIGUES, F. F. L. et al. Conhecimento e atitudes: componentes para a educação em diabetes. Revista Latino-Americana de Enfermagem, Ribeirão Preto, v. 17, n. 4, p. 28-34, jul./ago. 2009. 
SALOMÉ, G. M.; ESPÓSITO, V. H. C.; SILVA, G. T. R. Sentimentos vivenciados pelos pacientes diabéticos com lesão no pé e com o risco de amputação. Revista Nursing, São Paulo, v. 10, n. 116, p. 45-50, 2008.

SANTOS, M. A. et al. Representações sociais de pessoas com diabetes acerca do apoio familiar percebido em relação ao tratamento. Revista da Escola de Enfermagem, São Paulo, v. 45, n. 3, p. 651-658, jun. 2011.

SANTOS, M. S. Avaliação da adesão terapêutica de pacientes com diabetes mellitus tipo 2. 2010. 107 f. Dissertação (Mestrado em Ciências da Saúde) - Universidade Cruzeiro do Sul, São Paulo, 2010.

SCHUTZ, R. et al. Associação entre diferentes fatores de risco para doenças cardiovasculares e hipertensão arterial de pacientes com dislipidemia pertencentes a uma comunidade universitária. Revista Brasileira de Análises Clínicas, Florianópolis, v. 40, n. 2, p. 97-99, fev. 2008.

SILVESTRE, M. I. J. et al. Estratégias de coping na doença crônica. 2008. Disponível em: <http://portfoliodopedro.com/>. Acesso em: 21 out. 2011.

SOCIEDADE BRASILEIRA DE DIABETES (SBD). Cuidados de enfermagem em diabetes mellitus. São Paulo: SBD, 2009.

. Diabetes no idoso. 2011. Disponível em: <http://www.diabetes.org.br/>. Acesso em: 05 ago. 2011.

TRENTINI, M. et al. Enfrentamento de situações adversas e favoráveis por pessoas idosas em condições crônicas de saúde. Revista Latino-Americana de Enfermagem, Ribeirão Preto, v. 13, n. 1, p. 38-45, jan./fev. 2005.

VIVAN, A. S.; ARGIMON, I. I. L. Estratégias de enfrentamento, dificuldades funcionais e fatores associados em idosos institucionalizados. Cadernos de Saúde Pública, Rio de Janeiro, v. 25, n. 2, p. 436-444, fev. 2009. 